\title{
Imidacloprid Poisoning-Newer Insecticide and Fatal Toxicity
}

\author{
Ramya Iyyadurai • Ige Abraham George • \\ John Victor Peter
}

Published online: 16 March 2010

(C) American College of Medical Toxicology 2010

To the Editor,

There is a constant search for new and potent insecticides nontoxic to humans. We describe fatal toxicity with one such insecticide considered relatively safe.

A 34-year-old male from rural India was brought following the deliberate consumption of imidacloprid in a suicide attempt. At presentation, he was comatose with dilated nonreacting pupils, diaphoretic, and cyanosed. His blood pressure was unrecordable, and his heart rate was $50 / \mathrm{min}$. Following intubation and stabilization of hemodynamics with crystalloids, a gastric lavage was performed. Subsequently, he was referred to our hospital. On arrival, $2 \mathrm{~h}$ later, he continued to be unconscious with fixed dilated pupils. His blood pressure was $130 / 80 \mathrm{mmHg}$ and pulse rate $86 / \mathrm{min}$.

Following transfer to intensive care, he required epinephrine infusion to maintain blood pressure. Deterioration of renal function was noted from the second day with progressive oligoanuria (peak creatinine $6.9 \mathrm{mg} / \mathrm{dl}$ ) and severe metabolic acidosis necessitating dialysis. Creatinine phosphokinase levels of $67,980 \mathrm{U} / \mathrm{L}$ (reference $<170 \mathrm{U} / \mathrm{L}$ ) suggested rhabdomyolysis. Hospital stay was further complicated by the development of liver dysfunction, ventilator-associated pneumonia, and refractory septic shock. Despite appropriate supportive therapy, he succumbed on day 12 .

There was no funding for this report.

J. V. Peter $(\bowtie)$

Medical Intensive Care Unit,

Christian Medical College and Hospital,

Vellore, India

e-mail: peterjohnvictor@yahoo.com.au

R. Iyyadurai $\cdot$ I. A. George $\cdot$ J. V. Peter

Department of Medicine and Critical Care, Christian Medical

College and Hospital,

Vellore, India
Neonicotinoids, agonists at the nicotinic acetylcholine receptors (nAChRs), induce neuromuscular paralysis. The high selectivity for nAChRs (particularly the a4b2 subtype) in insects compared with mammals results in their favorable toxicological profile $[1,2]$. Imidacloprid, the commonest neonicotinoid used in South Asia, was recently reported to have a $0 \%$ case fatality in a series of 68 patients presenting with poisoning [3]. Despite their safety profile, other publications have described rhabdomyolysis [4], neuropsychiatric manifestations [4], ventricular fibrillation [5], and deaths with imidacloprid [5-7].

This patient manifested neurological dysfunction and rhabdomyolysis. The initial neurological dysfunction, probably due to central nicotinic stimulation, was compounded by ischemic and metabolic encephalopathy. Renal injury was perpetuated by rhabdomyolysis, hypotension, and toxin. Progressive deterioration and eventual demise was due to multiple organ dysfunction syndrome and not the toxin per se. Serum imidacloprid level would have helped elucidate causality, but was unavailable. This report of imidacloprid toxicity sensitizes clinicians to an emerging cause of poisoning and highlights the need for a careful review of its toxicity profile.

Conflict of interest The authors declare that they have no conflict of interest.

\section{References}

1. Tomizawa M, Casida JE (2005) Neonicotinoid insecticide toxicology: mechanisms of selective action. Annu Rev Pharmacol Toxicol 45:247-268 
2. Tomizawa M, Casida JE (2003) Selective toxicity of neonicotinoids attributable to specificity of insect and mammalian nicotinic receptors. Annu Rev Entomol 48:339-364

3. Mohamed F, Gawarammana I, Robertson TA, Roberts MS, Palangasinghe C, Zawahir S et al (2009) Acute human selfpoisoning with imidacloprid compound: a neonicotinoid insecticide. PLoS ONE 4:e5127, Epub 2009 Apr 8

4. Agarwal R, Srinivas R (2007) Severe neuropsychiatric manifestations and rhabdomyolysis in a patient with imidacloprid poisoning. Am J Emerg Med 25:844-845
5. Huang $\mathrm{NC}$, Lin SL, Chou $\mathrm{CH}$, Hung YM, Chung HM, Huang ST (2006) Fatal ventricular fibrillation in a patient with acute imidacloprid poisoning. Am J Emerg Med 24: 883-885

6. Proenca P, Teixeira H, Castanheira F, Pinheiro J, Monsanto PV, Marques EP et al (2005) Two fatal intoxication cases with imidacloprid: LC/MS analysis. Forensic Sci Int 153:7580

7. Shadnia S, Moghaddam HH (2008) Fatal intoxication with imidacloprid insecticide. Am J Emerg Med 26:634 